\title{
A MULTI-MODEL APPROACH TO SAINT-VENANT EQUATIONS: A STABILITY STUDY BY LMIS
}

\author{
VALÉRIE DOS SANTOS MARTINS, MICKAEL RODRIGUES, MAMADOU DIAGNE
}

\author{
Automatic and Process Control Laboratory (LAGEP) \\ University of Lyon 1, CNRS, UMR 5007, Villeurbanne F-69622, France \\ e-mail: dossantos@lagep.univ-lyon1.fr, mickael.rodrigues@univ-lyon1.fr
}

\begin{abstract}
This paper deals with the stability study of the nonlinear Saint-Venant Partial Differential Equation (PDE). The proposed approach is based on the multi-model concept which takes into account some Linear Time Invariant (LTI) models defined around a set of operating points. This method allows describing the dynamics of this nonlinear system in an infinite dimensional space over a wide operating range. A stability analysis of the nonlinear Saint-Venant PDE is proposed both by using Linear Matrix Inequalities (LMIs) and an Internal Model Boundary Control (IMBC) structure. The method is applied both in simulations and real experiments through a microchannel, illustrating thus the theoretical results developed in the paper.
\end{abstract}

Keywords: Saint-Venant equation, multi-model, LMIs, infinite dimensional system, exponential stability, strongly continuous semigroup, internal model boundary control.

\section{Introduction}

Control problems for irrigation channels are of economic and environment interest and considerable research has been conducted in this area. Indeed, water is a precious resource which has to be efficiently managed and protected. However, water losses in irrigation channels are substantial remaining the biggest consumer of fresh water $(\simeq 80 \%)$. The automation of irrigation channels has improved the output of such a process, but the losses are still around $30 \%$, due to inefficient management and control. In order to avoid overflows and to satisfy specific water requirements, the level of instrumentation (e.g., water level measurements and motor-driven gates) and automation in open channel networks increases (see the work of Mareels et al. (2005) for an overview). On the other hand, the demands, the ecological constraints and necessary limitations have become more and more important these last years. Instrumentation and control (e.g., by a state feedback) allow improving the management of such systems. Nevertheless, it is necessary to improve them so as to take into account in a more precise way every event that may occur.

In order to deliver water, it is important to ensure that the water level and the flow rate in the open channel remain at given values. The difficulty in this control system is that only the gate positions are able to meet performance specifications. That is why the use of boundary control laws satisfying the control objectives is required. Open surface channels possess nonlinear complex dynamics because they couple phenomena of transport and those of delay. Those distributed parameters systems have dynamics represented by hyperbolic SaintVenant Partial Differential Equations (PDEs). This problem has been previously considered in the literature using a wide variety of techniques (see the use of classical linear control theory by Malaterre et al. (1998), Papageorgiou and Messmer (1989), Weyer (2002)). Some of them take into account the uncertainties and apply some robust control approaches (e.g., Litrico and Georges, 1999). Other researchers have studied directly the nonlinear dynamics (Zaccarian et al., 2007; Litrico et al., 2005; Dulhoste et al., 2001; Dos Santos and Prieur, 2008). Recent approaches have considered the distributed nature of the system. Using Riemann's coordinate approach to SaintVenant equations, stability results are given by Greenberg and $\mathrm{Li} \mathrm{(1984)} \mathrm{for} \mathrm{a} \mathrm{system} \mathrm{of} \mathrm{two} \mathrm{conservation} \mathrm{laws} \mathrm{and}$ by $\mathrm{Li}$ (1994) for a system of a larger dimension. Lyapunov techniques have been used by Coron et al. (2007), Dos Santos et al. (2008) as well as Dos Santos and Prieur (2008).

In practice, process industries such as mining, chem- 
ical engineering, or water treatment processes are characterized by complex processes which often operate in multiple operating regimes (Blesa et al., 2010). It is often difficult to obtain nonlinear models that accurately describe plants in all regimes. Also, considerable effort is required for development of nonlinear models. Comparatively, different techniques for linear system identification, control and monitoring are available. An attractive alternative to nonlinear techniques is to use a multiple linear model strategy. The concept of multiple models, or 'multi-models', is based on the partitioning of the operating range of a system into separated regions by applying local linear control to each region (Murray-Smith and Johansen, 1997).

The multi-model structure is well adapted for nonlinear systems because it allows determining a set of linear models defined around some predefined operating points. Each local model (sub-model) is defined as a Linear Time Invariant (LTI) model dedicated to a specific operating point. The multi-model philosophy is based on weighting functions, which ensure the transition between the different local models. These functions represent the degree of validity of each local model. This degree is a function of the system inputs, outputs and time. The multimodel approach has often been used for the modelling and control of nonlinear systems (Porfirio et al., 2003; Athans et al., 2005) and for fault diagnosis (Bhagwat et al., 2003; Gatzke and Doyle, 2002; Rodrigues et al., 2008). Some authors refer to it as a gain scheduling strategy (Leith and Leithead, 2000), or Linear Parameter Varying (LPV) systems with the same formalism (Hamdi et al., 2011), or interpolated controllers (Banerjee et al., 1995) and switching controllers (Narendra et al., 1995).

The use of the multi-model representation for a stability study of systems described by nonlinear PDEs is something new in the literature on such systems, but some researchers like Wang et al. (2011) have recently developed a control strategy using Takagi-Sugeno models for nonlinear PDE systems with a stability study. More generally, common approaches are based on a finite dimensional approximation of the nonlinear PDE and adaptive control. The stability and the control of such systems in infinite dimensions are still an open problem.

In this paper, an analysis of the stability of the nonlinear Saint-Venant PDE is proposed through the use of the multi-model and Internal Model Boundary Control (IMBC) structures. The stability study is performed based on a Linear Matrix Inequality (LMI) due to the effectiveness in calculating a unique gain solution for multiple models (Lopez-Toribio et al., 1999; Rodrigues et al., 2007; Dos Santos Martins and Rodrigues, 2011).

The paper is organized as follows. Firstly, SaintVenant equations are presented along with the control problem. The internal model boundary control is explained and the physical constraints are given. Secondly, linearized systems are developed around a set of equilibria which depend on the space variable. Their insertion into the LMI formalism is also described. The third part of the paper is dedicated to the design of an integral feedback gain by an LMI which ensures the system stability: an integral controller is designed and implemented using a "Lyapunov/LMI" approach. The last section is dedicated to simulations and experiments. The data used are those of the water channel of Valence. Comparisons between initial experimental results using a PI-controller (done some years ago) and simulations with the presented integral controller using the theoretically tuned gain are realized. New experiments are implemented, too, with these theoretical gains found by LMI synthesis.

\section{Channel regulation problem statement}

Consider the class of water channels represented in Figs. 1 and 2, i.e., a reach of an open channel delimited by underflow and/or overflow gates, where

- $Q(x, t)$ is the water flow rate,

- $Z(x, t)$ is the height of water channel,

- $L$ is the length of the reach taken between the upstream $x_{u p}=0$ and the downstream $x_{d o}=L$,

- $U_{u p}=U_{0}(t), U_{d o}=U_{L}(t)$ are the openings of the gates at upstream and downstream.

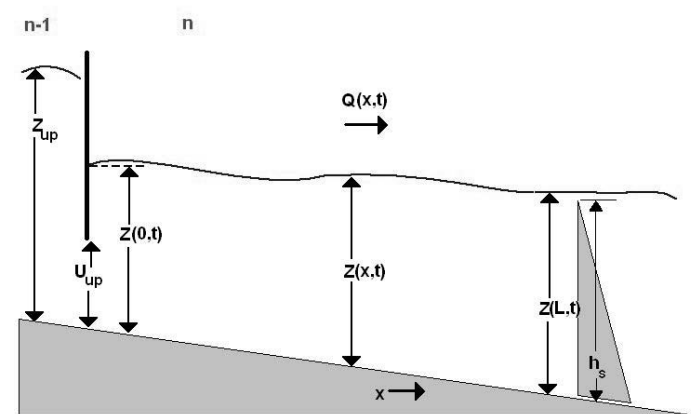

Fig. 1. Channel scheme: upstream underflow and downstream overflow gates.

The regulation problem concerns the stabilization of the water flow rate and/or the height of the water around an equilibrium for a reach denoted by $\left(Z_{e}(x), Q_{e}(x)\right)$. A linear model with varying coefficients can be deduced from the nonlinear PDE, in order to describe the variation in the water level and the flow for an open channel. Let us recall these models.

2.1. Model of a reach. The channel is supposed to have a sufficient length $L$ such that one can assume that the lateral movement is uniform. Nonlinear Saint-Venant 


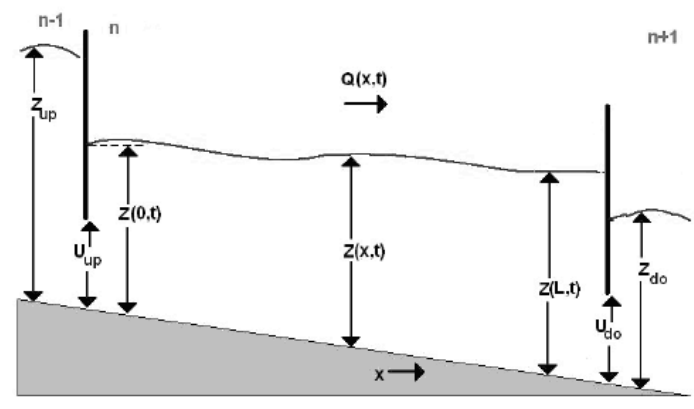

Fig. 2. Channel scheme: two underflow gates.

PDEs, which describe the flow on the channel, are the following (Georges, 2002):

$$
\begin{aligned}
\partial_{t} Z & =-\partial_{x} \frac{Q}{b}, \\
\partial_{t} Q & =-\partial_{x}\left(\frac{Q^{2}}{b Z}+\frac{1}{2} g b Z^{2}\right)+g b Z(I-J), \\
Z_{0}(x) & =Z(x, 0), \quad Q_{0}(x)=Q(x, 0),
\end{aligned}
$$

$\forall x \in \Omega=\left(x_{u p}, x_{d o}\right)=(0, L), t>0$, where $I$ is the slope, $b$ is the channel width, $g$ is the constant gravity.

Here $J$ is the friction slope from the ManningStrickler formula and $R$ is the hydraulic radius. $J$ and $R$ are defined such that

$$
J=\frac{n^{2} Q^{2}}{(b Z)^{2} R^{4 / 3}}, \quad R=\frac{b Z}{b+2 Z} .
$$

The different limit conditions allow us to consider two control cases, for all $x \in \Gamma=\partial \Omega$ :

Case A. Single variable control, spillway case.

The equation of the upstream condition of the reach $(x=$ $x_{u p}$ ) is given by

$$
Q\left(x_{u p}, t\right)=U_{u p}(t) \Psi_{1}\left(Z\left(x_{u p}, t\right)\right),
$$

with $\Psi_{1}(Z)=K_{1} \sqrt{2 g\left(Z_{u p}-Z\right)}$. The downstream condition of the reach $\left(x=x_{d o}\right)$ is given by the spillway equation, cf. Fig. 1:

$$
Z\left(x_{d o}, t\right)=\Psi_{2}\left(Q\left(x_{d o}, t\right)\right),
$$

where

$$
\Psi_{2}(Q)=\left(\frac{Q^{2}}{2 g K_{2}}\right)^{1 / 3}+h_{s} .
$$

$Z_{u p}$ is the water height at the upstream of the gate, $K_{i}$ is the product of the channel width and the water flow rate coefficient of the gate $n^{0} i, U_{u p}(t)$ is the upstream control. $h_{s}$ is the height of the spillway which considered constant in this case. Note that the controlled variable is the height $Z\left(x_{d o}\right)$. Thus, in this case $x_{u p}=0, x_{d o}=L, U_{u p}=U_{0}$, (cf. Fig. (1).
Case B. Multi-variable control.

The upstream condition equation is still (5). Another control can appear at the downstream of the reach, i.e., at $x=x_{d o}$ (Fig. 2):

$$
Q\left(x_{d o}, t\right)=U_{d o}(t) \Psi_{3}\left(Z\left(x_{d o}, t\right)\right),
$$

where $\Psi_{3}(Z)=K_{2} \sqrt{2 g\left(Z-Z_{d o}\right)}$ and $U_{d o}(t)$ is the downstream control of the reach, $Z_{d o}$ is the water height downstream of the gate (cf. Fig. 2). Note that

- The upstream and downstream depend on the reach considered. The same case is for the abscissa and the gates.

- Case B is considered, i.e., the multi-variable control case.

2.2. Regulation model. An equilibrium state $\left(\partial_{t}(\cdot) \equiv\right.$ 0 ) of the system satisfies the following equations:

$$
\begin{aligned}
& \partial_{x} Q_{e}=0, \\
& \partial_{x} Z_{e}=g b Z_{e} \frac{I-J_{e}}{g b Z_{e}-Q_{e}^{2} / b Z_{e}^{2}},
\end{aligned}
$$

Remark 1. The river case is considered and it follows that:

$$
Z_{e}>\sqrt[3]{Q_{e}^{2} /\left(g b^{2}\right)}
$$

Note that $Q_{e}$ is constant but $Z_{e}$ depends on the space variable. The linearized model around an equilibrium point $\left(Z_{e}(x) \quad Q_{e}(x)\right)^{t}$ is written down by introducing

$$
\xi(t)=(z(t) q(t))^{t} .
$$

We obtain

$$
\begin{aligned}
\partial_{t} \xi(x, t)= & A_{1}(x) \partial_{x} \xi(x, t)+A_{2}(x) \xi(x, t) \\
= & \mathcal{A}(x) \xi(x, t), \\
\xi(x, 0)= & \xi_{0}(x), \\
q\left(x_{u p}, t\right)= & U_{u p, e} \partial_{z} \Psi_{1}\left(Z_{e}\left(x_{u p}, t\right)\right) z\left(x_{u p}, t\right) \\
& +u_{u p}(t) \Psi_{1}\left(Z_{e}\left(x_{u p}, t\right)\right), \\
q\left(x_{d o}, t\right)= & U_{d o, e} \partial_{z} \Psi_{3}\left(Z_{e}\left(x_{d o}, t\right)\right) z\left(x_{d o}, t\right) \\
& +u_{d o}(t) \Psi_{3}\left(Z_{e}\left(x_{d o}, t\right)\right),
\end{aligned}
$$

where $U_{u p, e}$ and $U_{d o, e}$ are the opening gates for the upstream and downstream at the equilibrium and $u_{u p}(t)$, $u_{d o}(t)$ are the variations of these opening gates to be controlled. The matrices $A_{1}(x), A_{2}(x)$ are given by

$$
\begin{aligned}
& A_{1}(x)=\left(\begin{array}{cc}
0 & -a_{1} \\
-a_{2}(x) & -a_{3}(x)
\end{array}\right), \\
& A_{2}(x)=\left(\begin{array}{cc}
0 & 0 \\
a_{4}(x) & -a_{5}(x)
\end{array}\right),
\end{aligned}
$$


with

$$
\begin{aligned}
a_{1} & =1 / b, \\
a_{2}(x) & =g b Z_{e}(x)-\frac{Q_{e}^{2}}{b Z_{e}^{2}(x)}, \\
a_{3}(x) & =\frac{2 Q_{e}}{b Z_{e}(x)}, \\
a_{4}(x) & =g b\left(I+J_{e}(x)+\frac{\frac{4}{3} J_{e}(x)}{1+2 Z_{e}(x) / b}\right), \\
a_{5}(x) & =\frac{2 g b J_{e}(x) Z_{e}(x)}{Q_{e}} .
\end{aligned}
$$

The control problem is to find variations in $u_{u p}(t)$ at the extreme $x=x_{u p}$ and $u_{d o}(t)$ at the extreme $x=$ $x_{d o}$ of the reach such that the downstream water level, $z\left(x_{d o}, t\right)=z(L, t)$ (measured variables), tracks a reference signal $r(t)$.

The reference signal $r(t)$ is chosen for all cases: constant or non-persistent (a stable step response of a nonoscillatory system).

In this paper, the control scheme based on the Internal Model Boundary Control (IMBC) (Dos Santos, 2004; Dos Santos et al., 2005) is adopted as illustrated in Fig. 3. This control strategy integrates the process model in real time and allows regulating the water height in all the points of the channel by taking into account the error between the linearized model and the real system (or the nonlinear model for the simulations).

Observe that

- $M_{f}$ is a linear filtering model of a finite dimension which aims at filtering the error signal $e(t)=y_{s}(t)-$ $y(t)$.

- $M_{r}$ is a pursuit model which allows to impose dynamics by means of a fixed reference $r(t)$.

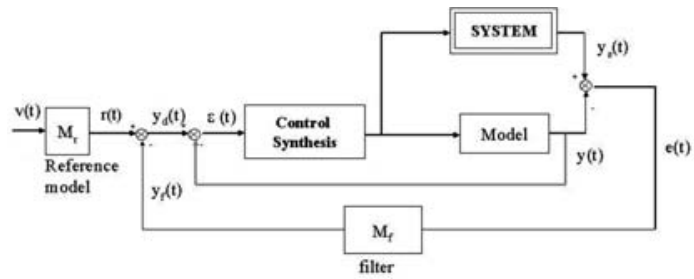

Fig. 3. IMBC structure.

2.3. Stability of the system. Equation (10) describes the dynamics of the open loop system. In this representation, the state vector $\xi(x, t)$ is not explicitly linked to the boundary control. In order to design an output feedback and to study the closed-loop stability, an operator $D$ of the boundary control distribution is introduced.
It is a bounded operator such that $\operatorname{Im}(D) \subset \operatorname{Ker}(A)$ and $D u \in D(\mathcal{A})$ and (Dos Santos, 2004; Touré and Rudolph, 2002; Sakawa and Matsushita, 1975)

$$
\xi(x, t)=\varphi(x, t)+D u(t) .
$$

This operator is naturally null in the domain of $A(x)$ as it is active only on the domain boundary. This change of variables allows obtaining a Kalman system representation (Touré and Rudolph, 2002; Sakawa and Matsushita, 1975; Alizadeh Moghadam et al., 2011):

$$
\begin{aligned}
\partial_{t} \varphi(x, t) & =\mathcal{A}(x) \varphi(x, t)-D \dot{u}, \\
\varphi(x, 0) & =\varphi_{0}(x)=\xi_{0}(x)-D u(0) .
\end{aligned}
$$

It has been proved that the open-loop system described below is exponentially stable (Dos Santos, 2004; Dos Santos and Toure, 2005), as the operator of the linearized system in infinite dimensions generates an exponentially stable $C_{0}$-semigroup. Moreover, under a PI control $u(t)=\alpha_{i} \kappa_{i} \int \varepsilon(s) \mathrm{d} s+\alpha_{p} \kappa_{p} \varepsilon(t) \in U=\mathbb{R}^{n}$, $u \in C^{\alpha}([0, \infty], U)^{1}$, conditions on the tuning parameters are also given to ensure the stability of the closedloop nonlinear system using the IMBC structure and the properties of stability of the closed-loop linearized system Fig. 3. For example, some of those conditions are taken for the tuned parameters of the PI-control:

$$
\begin{aligned}
& 0 \leq \alpha_{i}<\alpha_{i, \max }=\min _{\lambda \in \Gamma}\left(a\left\|R\left(\lambda ; \mathcal{A}_{e}\right)\right\|+1\right)^{-1}, \\
& 0 \leq \alpha_{p}<\alpha_{p, \max }=\left(\sup _{\lambda \in \Gamma} a\|R(\lambda ; \mathcal{A})\|\right)^{-1},
\end{aligned}
$$

where $\mathcal{A}_{e}$ is a part of the series development of the closedloop operator (Dos Santos and Toure, 2005), and $R(\lambda ; K)$ is the resolvent operator of $K$, while $a$ signifies a constant which depends on $\mathcal{A}_{e}$.

Those theoretical results were coupled with simulations and experiments which confirmed this approach (Dos Santos, 2004; Dos Santos and Toure, 2005). The experiments showed limitations due to the linearization around an equilibrium state and a first attempt at multimodel experiments was successfully realized (Fig. 10), but it was not optimal and no theoretical proof was given. The aim of this paper is to develop a first step towards this proof.

In order to control the water level over a wide operational range, a set of local models are considered around judicious operating points: each model is an approximation of the process in a small interval of the operating range and a control is synthesized and activated on this interval when the system goes through it. The idea here is to define necessary conditions to preserve the stability of this system all along the operating range.

\footnotetext{
${ }^{1}$ The regularity coefficient is generally taken as $\alpha=2$.
} 
2.4. Multi-model representation of Saint-Venant equations. The multi-model structure like that of Rodrigues et al. (2008), or Hamdi et al. (2011), allows controlling the system over a wide operating range because it takes into account the different sub-models which can be activated under different operating regimes (MurraySmith and Johansen, 1997). The representation of the (the Saint-Venant PDE) around $N$ operating points by the multi-models approach is defined by the following equations:

$$
\begin{aligned}
\partial_{t} \xi(x, t) & =\sum_{i=1}^{N} \mu_{i}(\zeta(t)) \mathcal{A}_{i}(x) \xi(x, t), \\
\mathcal{A}_{i}(x) & =A_{1, i}(x) \partial_{x}+A_{2, i}(x) \\
\xi_{0}(x) & =\xi(x, 0)
\end{aligned}
$$

where

- $\mathcal{A}_{i}(x)$ is the operator which corresponds to the $i$-th equilibrium state;

- $\zeta(t)$ is a function depending on some decision variables directly linked with the measurable state variables and eventually with the input;

- $\mu_{i}(\zeta(t))$ is the weighting function that based on the output height of the process $z_{L}$ and determining which sub-model is used for the control law.

A multi-model approach can be developed. It makes the study of the stability by the second Lyapunov method possible.

In the following paragraph, the synthesis of a control law by the LMI technique is outlined. An output feedback is considered under an integral control and the synthesis of the gain by the LMI technique ensures the system stability.

\section{Stability study by the LMI}

In this part, the closed-loop structure (Fig. 3) is studied under an integral feedback. The pursuit model $\left(M_{r}\right)$ and the filtering model $\left(M_{f}\right)$ are not considered. The choice of an integrator can be justified by the fact that the derivative of the control $\dot{u}$ appears in the state equation (16).

3.1. Closed-loop structure for an integral feedback. For a control with an output feedback, $K$ is defined as the gain, $u(t)=K \int \varepsilon(t) \mathrm{d} t$, and it follows that (Dos Santos, 2004)

$$
\begin{aligned}
\varepsilon(t) & =r(t)-y(t), \\
u(t) & =K \int[r(\tau)-y(\tau)] \mathrm{d} \tau,
\end{aligned}
$$

with $y(t)=C(\xi(x, t)+E q(x, t))$, where $E q(x, t)=$ $\sum_{i=1}^{N} \mu_{i}(\zeta(t))\left(z_{e, i}(x) q_{e, i}\right)^{t}$ is the equilibrium state and, for example, $C E q(x, t)=\sum_{i=1}^{N} \mu_{i}(\zeta(t)) z_{e, i}(L)$ if the aim is to regulate the water level at $x=L$. From Eqn. (15), one deduces that

$$
y(t)=C \varphi(x, t)+C E q(x, t)+C D u(t),
$$

and by substituting $y(t)$ into the control equation,

$$
\begin{aligned}
u(t)= & K \int[r(\tau)-C E q(x, \tau) \\
& -C \varphi(x, \tau)-C D u(\tau)] \mathrm{d} \tau
\end{aligned}
$$

which implies

$$
\dot{u}(t)=K[r(t)-C E q(x, t)-C \varphi(x, t)-C D u(t)] .
$$

From Eqn. (16), the closed-loop expression is

$$
\begin{aligned}
\partial_{t} \varphi(x, t) \\
=\sum_{i=1}^{N} \mu_{i}(\zeta(t))\left[\mathcal{A}_{i}(x) \varphi(x, t)-D K(r(t)\right. \\
\quad-C E q(x, t)-C \varphi(x, t)-C D u(t))] \\
=\sum_{i=1}^{N} \mu_{i}(\zeta(t))\left[\left(\mathcal{A}_{i}(x)+D K C\right) \varphi(x, t)\right. \\
\quad+D K(C D u(t)+C E q(x, t)-r(t))] .
\end{aligned}
$$

Define

$$
\tilde{K}=D K
$$

Equation (25) can be written as

$$
\begin{aligned}
\partial_{t} \varphi(x, t)= & \sum_{i=1}^{N} \mu_{i}(\zeta(t))\left[\left(\mathcal{A}_{i}(x)+\tilde{K} C\right) \varphi(x, t)\right. \\
& +\tilde{K}(C D u(t)+C E q(x, t)-r(t))] \\
= & \mathcal{M}_{i}(x, t) .
\end{aligned}
$$

The stability conditions are ensured by using a quadratic Lyapunov function as in the works of Rodrigues et al. (2007) and Hamdi et al. (2011) in order to guarantee the convergence of the water height to the reference $r(t)$ over the widest operating range.

\subsection{Stability study with a quadratic Lyapunov func-} tion. Consider

$$
V(\varphi(x, t), t)=\langle\varphi(x, t), P \varphi(x, t)\rangle,
$$

where $\langle\cdot, \cdot\rangle$ is the inner product under study. The multimodel representation of the linearized Saint-Venant PDE defined by Eqn. (27) is asymptotically stable if there exists a matrix/operator $P>0$ such that ${ }^{1}$

\footnotetext{
${ }^{1}$ We assume that $\partial_{t} \psi=\dot{\psi}$ whatever the function $\psi$
} 
(i) for finite-dimensional systems,

$$
\begin{aligned}
& \dot{V}(\varphi(x, t), t)<0, \\
& \quad \Leftrightarrow\langle\dot{\varphi}, P \varphi\rangle+\langle\varphi, P \dot{\varphi}\rangle<0 ;
\end{aligned}
$$

(ii) for infinite-dimensional systems,

$$
\langle\dot{\varphi}, P \varphi\rangle+\langle P \varphi, \dot{\varphi}\rangle=-\langle\varphi, \varphi\rangle .
$$

Remark 2. As stated previously, we want to give the first elements of a proof of the stability using the LMI technique usually defined for finite-dimensional systems but applied to infinite-dimensional systems in this paper. Hante and Sigalotti (2010) worked on the stability of switching systems in infinite dimensions and they are still working to link those mathematical results to the LMI approach. The tools for the LMI technique developed in infinite dimensions have not been found by the authors and we try to developed those making a parallel with known techniques. Wang et al. (2011) also used an LMI technique for the stability study.

The previous results, like in the example part, can be put in parallel with the following theorem (Curtain and Zwart, 1995).

Theorem 1. Suppose that $A$ is the infinitesimal generator of a $C_{0}$-semigroup $T(t)$ on a Hilbert space $Z$. Then $T(t)$ is exponentially stable if and only if there exists a positive operator $P \in \mathcal{L}$ such that (with $\dot{z}=A z$ )

$$
\langle A z, P z\rangle+\langle P z, A z\rangle=-\langle z, z\rangle, \quad \forall z \in Z .
$$

The main difference here between this stability result in finite and infinite dimensions is located in the inequality of the Lyapunov function for a finite-dimensional system and the equality for infinite dimensional ones. This equality complexity can be removed in some cases. For example, for operators with a compact resolvent (Triggiani, 1975; Dos Santos, 2004; Curtain and Zwart, 1995), and in this case the same inequality from finite dimension is a sufficient and necessary condition for the infinitedimensional case. Indeed, the inequality from finite dimension cannot be transposed directly in infinite dimensions because the spectral growth assumption is not satisfied in general (it is in a finite dimension), i.e., an operator $A$ generating a $C_{0}$-semigroup $T_{A}(t)$ satisfies the spectral growth assumption if

$$
\sup \{\mathcal{R}(\lambda) ; \lambda \in \sigma(A)\}=w_{0}(A):=\inf _{t>0} \frac{\left\|T_{A}(t)\right\|}{t} .
$$

Thus, if the spectral growth assumption is satisfied and there exists a positive operator $P \in \mathcal{L}$ such that (with $\dot{z}=A z)$

$$
\langle A z, P z\rangle+\langle P z, A z\rangle<0, \quad \forall z \in Z,
$$

then $T(t)$ is exponentially stable.
For Saint-Venant equations, it has been shown that the operator has a compact resolvent (Dos Santos and Toure, 2005; Dos Santos, 2004), so it satisfies the spectral growth assumption.

Then, taking account of (27)-(32), it follows that one has to prove the inequality

$$
\left\langle\mathcal{M}_{i}, P \varphi\right\rangle+\left\langle\varphi, P \mathcal{M}_{i}\right\rangle<0 .
$$

The development of this inequality leads us to considering an inequality for each sub-system of index $i$ such that

$$
\begin{aligned}
& \left\langle\left[\mathcal{A}_{i}(x)+\tilde{K} C\right] \varphi(x, t), P \varphi(x, t)\right\rangle \\
& \quad+\langle\tilde{K}(C D u(t)-r(t)+C E q(x, t)), P \varphi(x, t)\rangle \\
& \quad+\langle\varphi(x, t), P \tilde{K}(C D u(t)-r(t)+C E q(x, t))\rangle \\
& \quad+\left\langle\varphi(x, t), P\left[\mathcal{A}_{i}(x)+\tilde{K} C\right] \varphi(x, t)\right\rangle<0 .
\end{aligned}
$$

In the inequality (34), which defines the system stability condition, the control parameter $u$ appears and it is a difficulty for the design of the gain $\tilde{K}$. Let us consider the following equality deduced from (15):

$$
C D u(t)-r(t)=C \xi(x, t)-r(t)-C \varphi(x, t) .
$$

Proposition 1. If there exists a positive definite matrix $P$, a matrix $W$ and a scalar $\alpha$ such that the following statements hold true:

$$
\begin{aligned}
& \text { (a) }\langle\varphi, P \widetilde{K}(C D u(t)+C E q(x, t)-r(t))\rangle \\
& \leq \alpha \varphi^{T} P \widetilde{K} C \varphi \\
& \text { (b) } \mathcal{A}_{i}^{T} P+P \mathcal{A}_{i}+W C+C^{T} W^{T}<0 \text {, }
\end{aligned}
$$

with

$$
\tilde{K}=\frac{1}{1+\alpha} P^{-1} W
$$

then the system (16) with an integral control input (23) is stable.

Proof. Let us consider the quadratic Lyapunov function

$$
V(\varphi(x, t), t)=\langle\varphi, P \varphi\rangle=\varphi^{T} P \varphi .
$$

Then one can write $\dot{V}(t)<0$ such that (34) can be upper bounded. Indeed, the inequality (36) implies that

$$
\begin{aligned}
\varphi^{T} P[ & \left.\left(\mathcal{A}_{i}+\widetilde{K} C\right) \varphi+\widetilde{K}(C D u-r)\right] \\
& \leq \varphi^{T} P\left[\left(\mathcal{A}_{i}+\widetilde{K} C\right) \varphi+\alpha \widetilde{K} C \varphi\right] \\
& \leq \varphi^{T} P\left[\mathcal{A}_{i} \varphi+\widetilde{K} C \varphi(1+\alpha)\right] .
\end{aligned}
$$

Hence, with the last consideration, the inequality (34) can 
be then upper bounded by

$$
\begin{aligned}
\varphi^{T}(x, & t)\left[\mathcal{A}_{i}(x)+\tilde{K} C\right]^{T} P \varphi(x, t) \\
& +\varphi^{T}(x, t) P\left[\mathcal{A}_{i}(x)+\tilde{K} C\right] \varphi(x, t) \\
& +[\tilde{K}(C D u(t)-r(t)+C E q(x, t))]^{T} P \varphi(x, t) \\
& +\varphi^{T}(x, t) P[\tilde{K}(C D u(t)-r(t)+C E q(x, t))] \\
\leq & \varphi^{T}(x, t)\left\{\left[\mathcal{A}_{i}(x)+(1+\alpha) \tilde{K} C\right]^{T} P\right. \\
& \left.+P\left[\mathcal{A}_{i}(x)+(1+\alpha) \tilde{K} C\right]\right\} \varphi(x, t) \\
=\varphi^{T} & {\left[\mathcal{A}_{i}^{T} P+P \mathcal{A}_{i}+W C+C^{T} W^{T}\right] \varphi<0 }
\end{aligned}
$$

with

$$
\tilde{K}=\frac{1}{1+\alpha} P^{-1} W
$$

Now, let us examine the inequality (36):

$$
\varphi^{T} P \widetilde{K}(C D u(t)+C E q(x, t)-r(t)) \leq \alpha \varphi^{T} P \widetilde{K} C \varphi
$$

Let us remember that the system is exponentially stable in open loop and for a PI-controller in closed loop, with gains correctly tuned (Dos Santos, 2004; Dos Santos and Toure, 2005) for a time $t$ well chosen, so one can assume that there exists $k>0$ such that

$$
|C \xi(x, t)+C E q(x, t)-r(t)| \leq k|C \varphi(x, t)| .
$$

Let $\varepsilon_{f(t)}=\operatorname{sign}(f(t))$. Then

$$
\begin{aligned}
& C D u(t)-(r(t)-C E q(x, t)) \\
& \quad=C \xi(x, t)-(r(t)-C E q(x, t)) \\
& \quad-C \varphi(x, t), \\
& |C D u-r+C E q(x, t)| \\
& \leq|C \xi-(r-C E q)|+|C \varphi| .
\end{aligned}
$$

That is to say, one can bound $(C D u-r)$ by

$$
\begin{aligned}
& -(k+1) \varepsilon_{(C \varphi)} C \varphi \\
& \quad \leq C D u-r \\
& \quad \leq(k+1) \varepsilon_{(C \varphi)} C \varphi, \\
& -(k+1) \varepsilon_{(C \varphi) \varepsilon_{\left(\varphi^{T} P \tilde{K}\right)} \varphi^{T} P \tilde{K} C \varphi} \\
& \quad \leq \varphi^{T} P \tilde{K}(C D u-r) \\
& \quad \leq(k+1) \varepsilon_{(C \varphi)} \varepsilon_{\left(\varphi^{T} P \tilde{K}\right)} \varphi^{T} P \tilde{K} C \varphi
\end{aligned}
$$

and thus

$$
\varphi^{T} P \tilde{K}(C D u-r) \leq(k+1) \varepsilon_{\left(\varphi^{T} P \tilde{K} C \varphi\right)} \varphi^{T} P \tilde{K} C \varphi .
$$

Accordingly, the inequality (36) is proved and

$$
\begin{aligned}
& \varphi^{T} P\left[\mathcal{A}_{i}+\tilde{K} C\right] \varphi+\varphi^{T} P[\tilde{K}(C D u-r) \varphi \\
& \quad \leq \varphi^{T} P\left[\mathcal{A}_{i}+\left(1+(k+1) \varepsilon_{\left(\varphi^{T} P \tilde{K} C \varphi\right)}\right) \tilde{K} C\right] \varphi
\end{aligned}
$$

Then, we obtain the gain $\tilde{K}$ as

$$
\begin{aligned}
& \varphi^{T} P\left[\mathcal{A}_{i}+\tilde{K} C\right] \varphi+\varphi^{T} P[\tilde{K}(C D u-r) \varphi \\
& \left\{\begin{aligned}
\leq & \varphi^{T} P\left[\mathcal{A}_{i}+(k+2) \tilde{K} C\right] \varphi \\
& \text { if } \varepsilon_{\left(\varphi^{T} P \tilde{K} C \varphi\right)}=1, \text { with } \alpha=k+1, \\
\leq & \varphi^{T} P\left[\mathcal{A}_{i}+k \tilde{K} C\right] \varphi \\
& \text { if } \varepsilon_{\left(\varphi^{T} P \tilde{K} C \varphi\right)}=-1, \text { with } \alpha=-k .
\end{aligned}\right.
\end{aligned}
$$

Remark 3. The solution of Proposition 1 may lead to a conservatism since a unique gain has been determined for all the models. However, it is here a first attempt into infinite dimensions and the use of the LMI applied to such PDE systems is very recent.

The gain $\tilde{K}$ has been implemented in the discretized model for simulations so as to verify the stability of the system. Some results have been obtained for a single reach with two underflow gates. The aim is to compare the simulation and experimental curves obtained with this method and those obtained experimentally by Dos Santos Martins in her previous works (Dos Santos, 2004; Dos Santos et al., 2005).

\section{Simulation and experimentation results}

Firstly, let us describe the benchmark used for the simulations and the experimentations which are presented in the second and third subsections, respectively.

4.1. Configuration and data of the channel. An experimental validation has been performed on the Valence micro-channel, cf. Figs. 4 and 5, Table 1. This pilot channel is located at the ESISAR ${ }^{2} / \mathrm{INPG}^{3}$ engineering school in Valence (France). It is operated under the responsibility of the LCIS ${ }^{4}$ laboratory. This experimental channel (total length of 8 meters) has an adjustable slope and a rectangular cross-section (a width of 0.1 meter). The channel is ended at its downstream by a variable overflow spillway and equipped with three underflow control gates (Figs. 4 and 5). Ultrasound sensors provide water level measurements at different locations of the channel (Fig. 6).

Note that the water flow is deduced from the gate equations and was not measured directly. For all numerical simulations, the Chang and Cooper theta-scheme of order 2 is used (Cordier et al., 2004). To validate this numerical discretization, comparisons between the numerical simulations with real data and those using the Preissmann scheme (which is employed in other works dealing with the control of flows (Litrico and Georges, 1999; Ouarit

\footnotetext{
${ }^{2}$ École Supérieure d'Ingénieurs en Systèmes Industriels Avancés Rhône-Alpes.

${ }^{3}$ Institut National Polytechnique de Grenoble.

${ }^{4}$ Laboratoire de Conception et d'Intégration des Systèmes.
} 


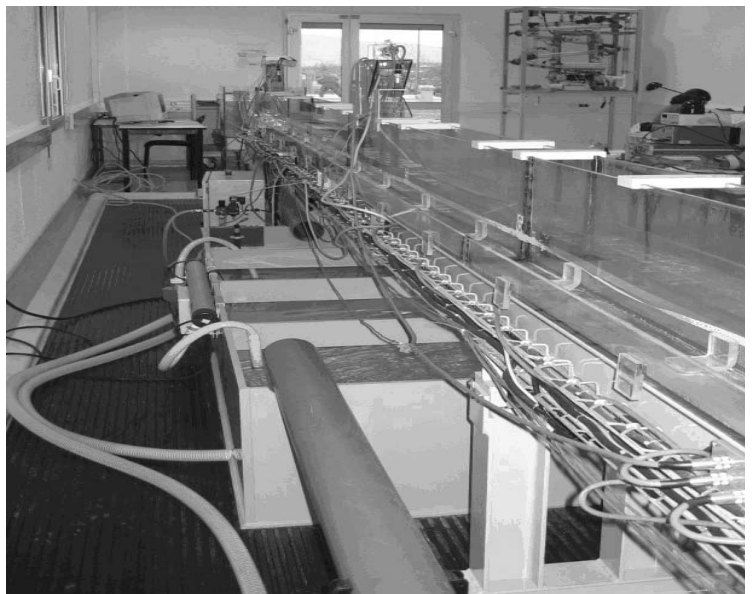

Fig. 4. Pilot channel of Valence.

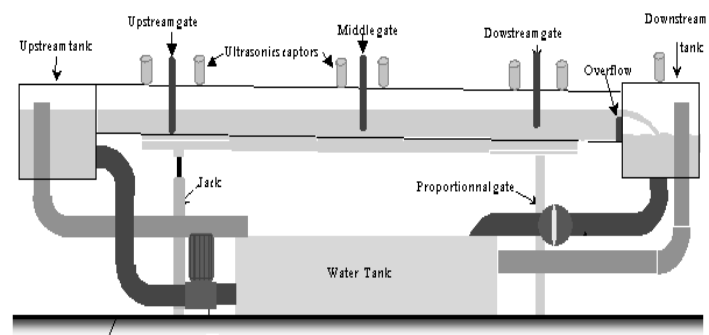

Fig. 5. Pilot channel of Valence.

et al., 2003)) were provided by Dos Santos and Prieur (2008). They validated the numerical discretization and the identification of the parameters. It also was done for the micro-channel (Fig. 7). The experimental data depicted below were filtered to get a better idea of the experimentation results. One reach has a length of $0.7 \mathrm{~m}$, the water level at the upstream of the first gate is $Z_{u p}=0.172$ $\mathrm{m}$ and at the downstream of the second gate $Z_{d o}=0.085$ $\mathrm{m}$ (theoretical values).

For this study, the following set of parameters from the practical Valence channel (Fig. 5) is considered;

- $n=20$ is the number of the discretizated points,

- $Z_{L}$ is the water height to be regulated.

Table 1. Parameters of the channel of Valence.

\begin{tabular}{|c||c|c|c|}
\hline Parameters & $B[\mathrm{~m}]$ & $L[\mathrm{~m}]$ & $K[\mathrm{~m}]\left[\mathrm{m}^{1 / 3} \cdot \mathrm{s}^{-1}\right]$ \\
\hline Values & 0.1 & 7 & 97 \\
\hline Parameters & $\mu_{0}$ & $\mu_{L}$ & slope $\left[\mathrm{m} \cdot \mathrm{m}^{-1}\right]$ \\
\hline Values & 0.6 & 0.73 & $1.6 \% 00$ \\
\hline
\end{tabular}

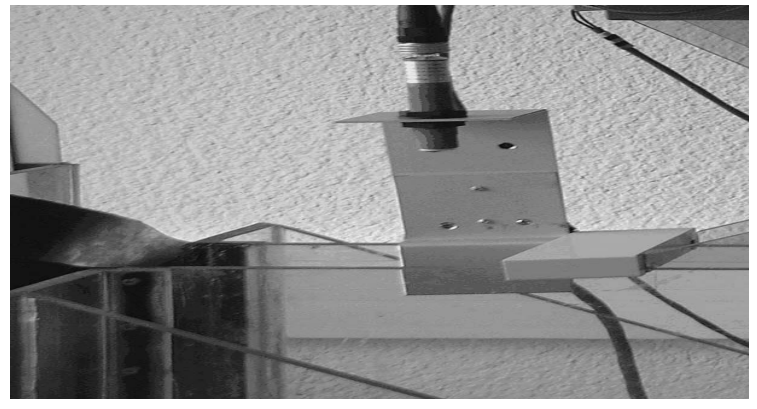

Fig. 6. Pilot channel of Valence: gate and ultrasound sensors.

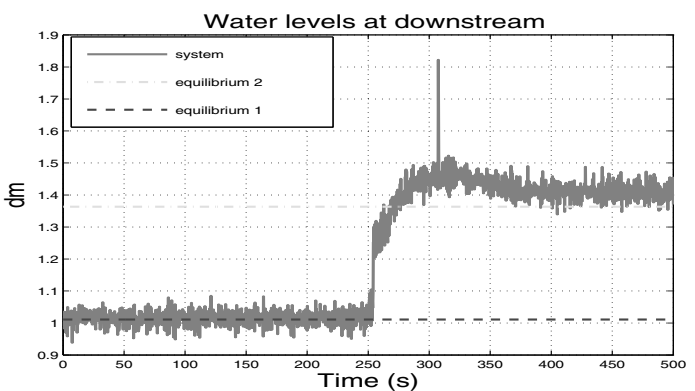

Fig. 7. Pilot channel of Valence: open-loop identification.

In this single reach with two gates, the regulation of the water height $Z_{L}$ at $x=L$ is done by controlling the openings $U_{0}(t)$ and $U_{L}(t)$ of the gates at upstream and downstream, respectively: it is a multi-variable control (cf. Fig. 2).

The equilibria profiles were chosen such that the calculated control law from the local models can be efficient over the entire operating range of the water height (Dos Santos, 2004). Let notice that it was experimentally verified that a local model is valid for around $\pm 20 \%$ of a water level equilibrium profile, i.e., the model and the data have the same behavior and values. In order to assign references which are included between $0.06 \mathrm{~m}$ and $0.2 \mathrm{~m}$, the operating points at $x=0$ are given in Table 2 .

Table 2. Initial set points for the simulations and the experimentations.

\begin{tabular}{|c|c|c|c|}
\hline \multicolumn{2}{|c|}{ Simulations } & \multicolumn{2}{c|}{ Experimentations } \\
\hline \hline$z_{e 1}(x=0)$ & $0.062 \mathrm{~m}$ & & \\
$z_{e 2}(x=0)$ & $0.077 \mathrm{~m}$ & $z_{e 1}(x=0)$ & $0.062 \mathrm{~m}$ \\
$z_{e 3}(x=0)$ & $0.099 \mathrm{~m}$ & $z_{e 2}(x=0)$ & $0.094 \mathrm{~m}$ \\
$z_{e 4}(x=0)$ & $0.135 \mathrm{~m}$ & $z_{e 3}(x=0)$ & $0.141 \mathrm{~m}$ \\
$z_{e 5}(x=0)$ & $0.18 \mathrm{~m}$ & & \\
\hline
\end{tabular}

The efficiency of the computer managing the DSpace card cannot bear more than three equilibrium states as it runs on a Windows 95 version. Consequently, their 
numbers towards the simulations had to be reduced.

In this application, the weighting function $\mu_{i}(\zeta(t))$ is equal to 1 if the output's height is included into the validity domain of the model, otherwise it is equal to 0 . The parameter $\zeta(t)$ exclusively depends on the output, which is the only one decision variable in this case.

4.2. Simulations. These results are obtained from an IMBC control and a multi-model approach with an LMI gain calculated in the previous section. Figure 8 shows that the output $Z(L)$ converges to the reference even if it strongly varies (variations $>100 \%$ ). The reference tracks slow dynamics and one can see that the convergence of the output is good.

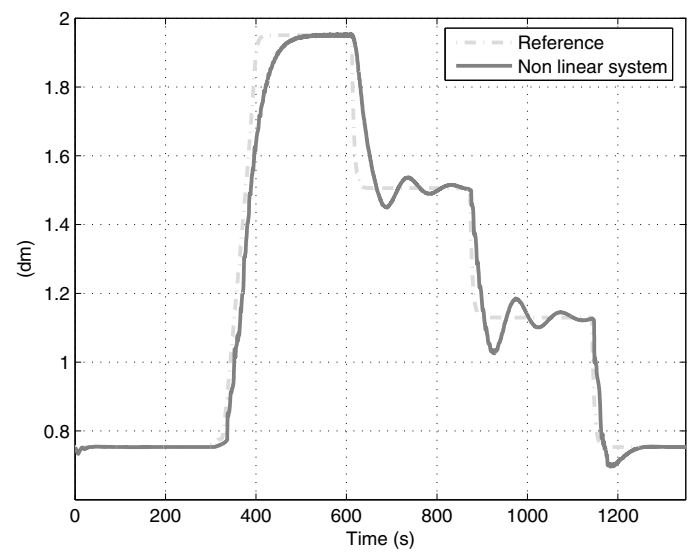

Fig. 8. Variations in the reference greater than $100 \%$.

The curves that describe the upstream and downstream gate openings of the reach are given in Fig. 9. The convergence of the output to the reference is ensured even when the reference is decreasing or increasing.

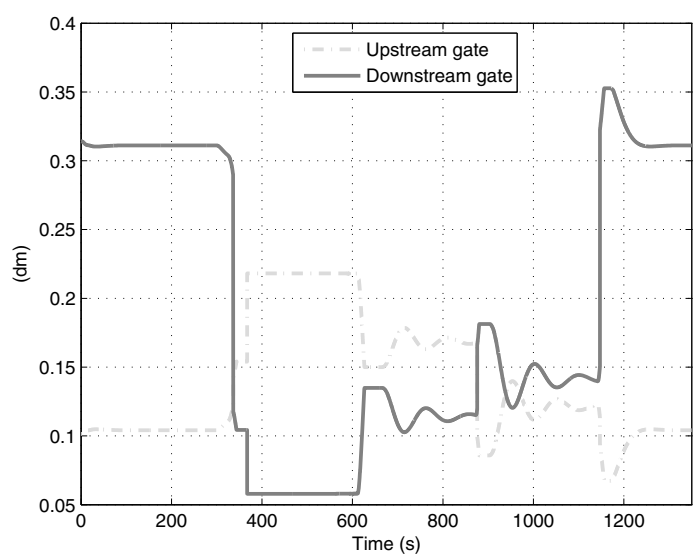

Fig. 9. Gate opening.

Next simulations are a first comparison between simulations using the theoretical gain obtained through the
LMI approach with the first tests realized some years ago by Dos Santos et al. (2005), using an experimental multimodel gain, without any theoretical study. Figure 10 represents the dynamic evolution of the simulated system and the experimental data. Note that the reference curves are equals, the experimental one stands for the signal $v(t)$, and the simulation one stands for the signal $r(t)$ after the reference model $M_{r}$ (cf. Fig. 3). Figure 11 compares the dynamics of the gate openings. The dynamics of the gates and the water level are similar and this result are promising. One can observe in Fig. 10 that the convergence is

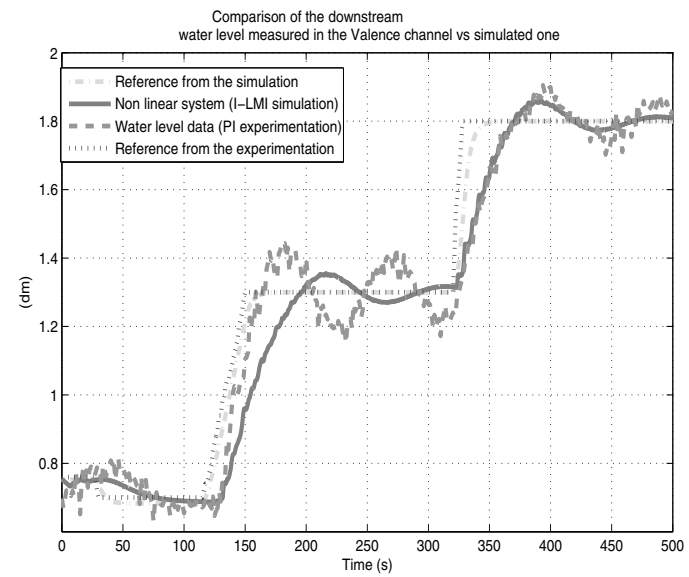

Fig. 10. Comparison of the downstream water level measured in the Valence channel with the first multi-models approach in 2004 vs. the simulated one with the LMI approach.

better than the one obtained experimentally and the overshoot is smaller, too. But the rising time is obviously long (at time $t=120-200 \mathrm{~s}$ example given) with the integral controller (simulation) vs. the PI-controller (experimentation). The next step is to design a new PI-controller using the LMI, to compare it with the experimental PI. It is presently under study.

Remark 4. The reference level of the channel is limited by physical constraints: the minimum is obtained with the maximum between the water height of the downstream reach and the fluvial condition, obtained from the initial model (9). The maximum from the size of the channel itself. In these simulations, the critical water height from fluvial constraint is $z_{e c}=0.0369 \mathrm{~m}$.

4.3. Experiments. The experiments were realized in the Valence channel (Fig. 5) with a multi-model approach and a gain calculated via the LMI approach. In the experimental figures (12)-(13), a wide range of the accessible water level is tested. Let us remark that some problems appear with the gates as sometimes they stay jammed because of the friction (e.g., here at time $t=50 \mathrm{~s}, t=120$ 

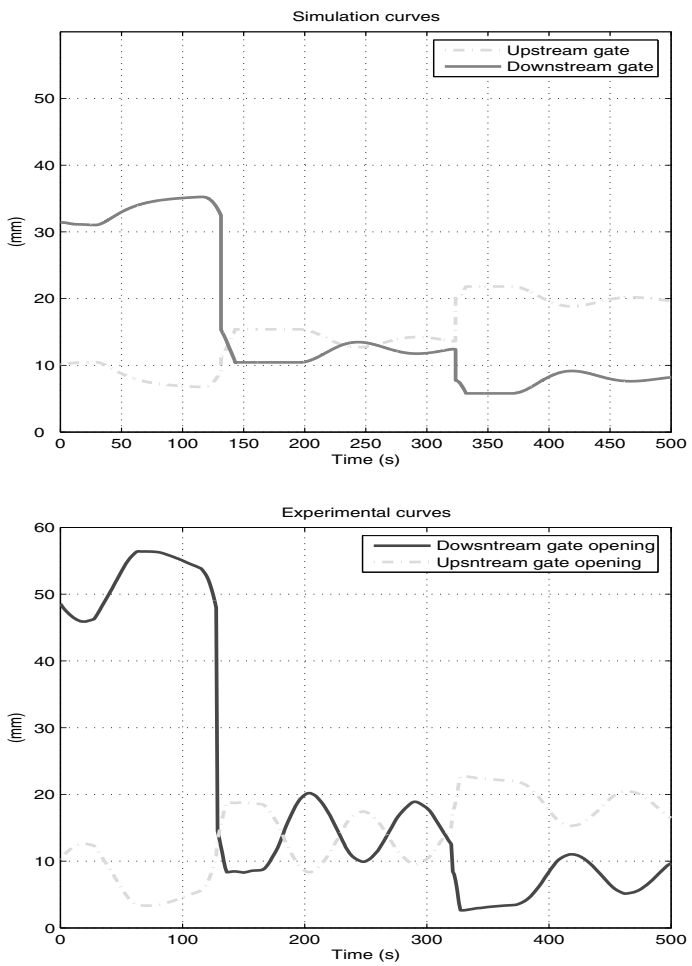

Fig. 11. Gate opening.

$\mathrm{s}, t=550 \mathrm{~s}$ and $t=625 \mathrm{~s}$ ). Those problems act like perturbations and the integral controller tries to compensate them.

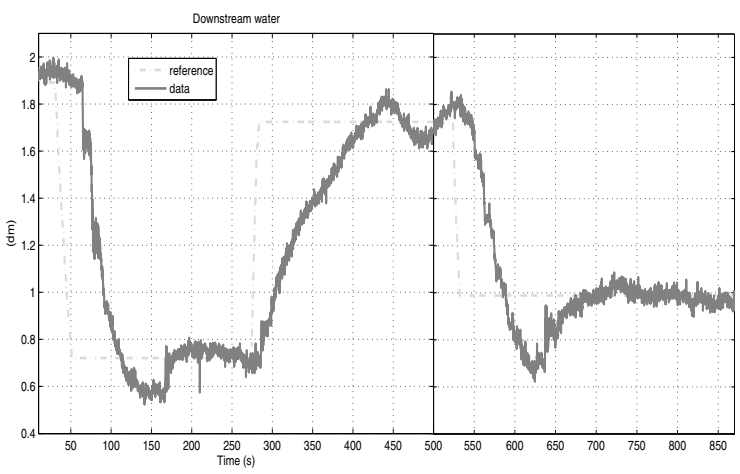

Fig. 12. Valence micro channel: downstream water level.

The convergence of the downstream water level is ensured in spite of the perturbations, but it is necessary to improve the controller to take them into account.

Those experimental results are relevant and promising for the applicability of our approach. Nevertheless, some improvements have to be done and the next step is to extend first results to a PI-controller, and thus to get robustness.

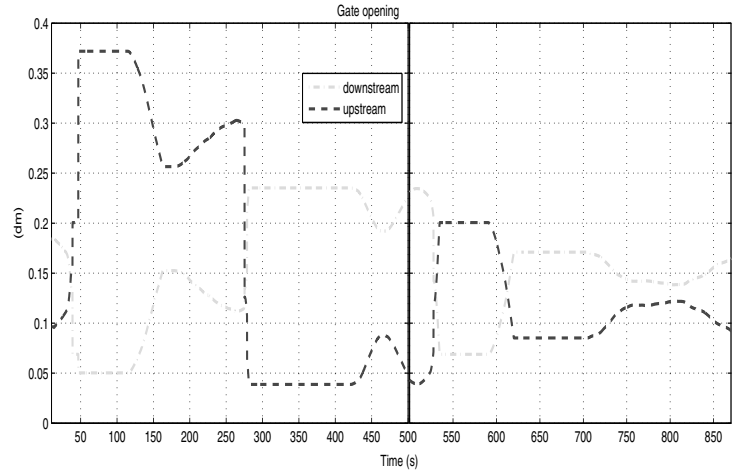

Fig. 13. Valence micro channel: gate openings.

\section{Conclusion}

First attempts to employ a multi-model approach for irrigation channels control, through an IMBC structure, have been made some years ago (Dos Santos, 2004; Dos Santos et al., 2005). Good experimental results, but with no attendant theory, were obtained and are promising. In this paper, the authors have formalized an LMI approach to the problem and gave first theoretical results in order to tune the new feedback gain trough LMI in the case of an integral controller. Simulations demonstrated the improvements realized towards the initial multi-model approach and new experiments confirmed the new theoretical gain tuning method. This paper allowed to find some stability results for infinite dimensional systems with LMI tools for finite dimensional systems.

Extensions to a PI-controller are actually investigated and preliminary results have been published (Dos Santos Martins and Rodrigues, 2011). The complexity is here located in the fact that it is a boundary control problem. Consequently, the control appears in a derivative form. Further experiments are planned for a new PI-controller and thus comparisons with the same controller have already been performed.

\section{Acknowledgment}

Thanks go to Professor E. Mendes and LCIS for allowing us to conduct our experimentations on the micro-channel.

\section{References}

Alizadeh Moghadam, A., Aksikas, I., Dubljevic, S. and Forbes, J. (2011). LQR control of an in finite dimensional timevarying CSTR-PFR system, 18th IFAC World Congress, Milan, Italy.

Athans, M., Fekri, S. and Pascoal, A. (2005). Issues on robust adaptive feedback control, Proceedings of the 16th IFAC World Congress, Prague, Czech Republic.

Banerjee, A., Arkun, Y., Pearson, R. and Ogunnaike, B. (1995). $H_{\infty}$ control of nonlinear processes using multiple linear 
models, Proceedings of the European Control Conference, Rome, Italy, pp. 2671-2676.

Bhagwat, A., Srinivasan, R. and Krishnaswamy, P.R. (2003). Multi-linear model-based fault detection during process transitions, Chemical Engineering Science 58: 1649-1670.

Blesa, J., Puig, V. and Bolea, Y. (2010). Fault detection using interval LPV models in an open-flow canal, Control Engineering and Practice 18(5): 460-470.

Cordier, S., Buet, C. and Dos Santos, V. (2004). A conservative and entropy scheme for a simplified model of granular media, Transport Theory and Statistical Physics 33(2): HYKE 2003-021.

Coron, J. M., d'Andréa Novel, B. and Bastin, G. (2007). A strict Lyapunov function for boundary control of hyperbolic systems of conservation laws, IEEE Transactions on Automatic Control 52(1): 2-11.

Curtain, R. and Zwart, H. (1995). An Introduction to Infinite Dimensional Linear Systems, Springer Verlag, New York, NY.

Dos Santos Martins, V. and Rodrigues, M. (2011). A proportional integral feedback for open channels control trough LMI design, 18th IFAC World Congress, Milan, Italy.

Dos Santos, V. (2004). Contrôle Frontière par Modèle Interne de Systèmes Hyperboliques: Application à la Régulation de Canaux d'Irrigation, Ph.D. thesis, University of Orléans, Orléans.

Dos Santos, V., Bastin, G., Coron, J.-M. and d'Andréa Novel, B. (2008). Boundary control with integral action for hyperbolic systems of conservation laws: Lyapunov stability analysis and experimental validation, Automatica 44(5): 1310-1318.

Dos Santos, V. and Prieur, C. (2008). Boundary control of open channels with numerical and experimental validations, IEEE Transactions on Control Systems Technology 16(99): 1252-1264.

Dos Santos, V. and Toure, Y. (2005). Irrigation multireaches regulation problem by internal model boundary control, 44th CDC-ECC05, IEEE Control Systems Society Conference, Seville, Spain, pp. 1905-1910.

Dos Santos, V., Toure, Y., Mendes, E. and Courtial, E. (2005). Multivariable boundary control approach by internal model, applied to irrigations canals regulation, Proceedings of the 16th IFAC World Congress, Prague, Czech Republic.

Dulhoste, J.-F., Besançon, G. and Georges, D. (2001). Nonlinear control of water flow dynamics by input-output linearisation based on a collocation model, European Control Conference, Porto, Portugal.

Gatzke, E. and Doyle, F. (2002). Use of multiple models and qualitative knowledge for on-line moving horizon disturbance estimation and fault diagnosis, Journal of Process Control 12(2): 339-352.

Georges (2002). Automatique pour la Gestion des Ressources en Eau, Edts IC2, Systèmes automatisés, Hermès, Paris.
Greenberg, J.-M. and Li, T. (1984). The effect of boundary damping for the quasilinear wave equations, Journal of Differential Equations 52(1): 66-75.

Hamdi, H., Rodrigues, M., Mechmeche, C., Theilliol, D. and Braiek, N. B. (2011). Fault detection and isolation in linear parameter varying descriptor systems via proportional integral observer, International Journal of Adaptive Control and Signal Processing 26(3): 224-240, DOI: 10.1002/acs. 1260 .

Hante, F. and Sigalotti, M. (2010). Existence of common Lyapunov functions for infinite-dimensional switched linear systems, 49th IEEE Conference on Decision and Control $(C D C)$, Atlanta, GA, USA, pp. 5668-5673.

Leith, D.J. and Leithead, W.E. (2000). Survey of gainscheduling analysis and design, International Journal of Control 73(11): 1001-1025.

Li, T. (1994). Global Classical Solutions for Quasilinear Hyperbolic Systems, Research in Applied Mathematics, Masson and Wiley, Paris/Milan/Barcelona.

Litrico, X., Fromion, V., Baume, J.-P., Arranja, C. and Rijo, M. (2005). Experimental validation of a methodology to control irrigation canals based on Saint-Venant equations, Control Engineering Practice 13(11): 1425-1437.

Litrico, X. and Georges, D. (1999). Robust continuous-time and discrete-time flow control of a dam-river system, I: Modelling, Journal of Applied Mathematical Modelling 23(11): 809-827.

Lopez-Toribio, C., Patton, R. and Daley, S. (1999). A mutiplemodel approach to fault-tolerant control using TakagiSugeno fuzzy modelling: Real application to an induction motor drive system, European Control Conference, ECC 99, Karlsruhe, Germany.

Malaterre, P.-O., Rogers, D. and Schuurmans, J. (1998). Classification of canal control algorithms, Journal of Irrigation and Drainage Engineering 124(1): 3-10.

Mareels, I., Weyer, E., Ooi, S., Cantoni, M., Li, Y. and Nair, G. (2005). Systems engineering for irrigation systems: Successes and challenges, Annual Reviews in Control 29(2): 191-204.

Murray-Smith, R. and Johansen, T. (1997). Multiple Model Approaches to Modelling and Control, Taylor and Francis, London.

Narendra, K., Balakrishnan, J. and Kermal, M. (1995). Adaptation and learning using multiple models, switching and tuning, IEEE Control Systems 15(3): 37-51.

Ouarit, H., Lefevre, L. and Georges, D. (2003). Robust optimal control of one-reach open channels, European Control Conference 03, Cambridge, UK.

Papageorgiou, M. and Messmer, A. (1989). Flow control of a long river stretch, Automatica 25(2): 177-183.

Porfirio, C. R., Neito, E. A. and Odloak, D. (2003). Multi-model predictive control of an industrial $\mathrm{c} 3 / \mathrm{c} 4$ splitter, Control Engineering Practice 11(7): 765-779. 
Rodrigues, M., Theilliol, D., Aberkane, S. and Sauter, D. (2007). Fault tolerant control design for polytopic LPV systems, International Journal of Applied Mathematics and Computer Science 17(1): 27-37, DOI: 10.2478/v10006-0070004-5.

Rodrigues, M., Theilliol, D., Adam-Medina, M. and Sauter, D. (2008). A fault detection and isolation scheme for industrial systems based on multiple operating models, Control Engineering Practice 16(2): 225-239.

Sakawa, Y. and Matsushita, T. (1975). Feedback stabilization of a class of distributed systems and construction of a state estimator, IEEE Transactions on Automatic Control AC-20 (6): 748-753

Touré, Y. and Rudolph, J. (2002). Controller design for distributed parameter systems, Encyclopedia of LIFE Support on Control Systems, Robotics and Automation, Vol. I, Eolss Publishers, Oxford, pp. 933-979.

Triggiani, R. (1975). On the stability problem in Banach space, Journal of Mathematical Analysis and Applications 52(3): 383-403.

Wang, J.-W., Wu, H.-N. and Li, H.-X. (2011). Distributed fuzzy control design of nonlinear hyperbolic PDE systems with application to nonisothermal plug-flow reactor, IEEE Transactions on Fuzzy Systems 19(3): 514-526.

Weyer, E. (2002). Decentralised PI controller of an open water channel, 15th IFAC World Congress, Barcelona, Spain.

Zaccarian, L., Li, Y., Weyer, E., Cantoni, M. and Teel, A.R. (2007). Anti-windup for marginally stable plants and its application to open water channel control systems, Control Engineering Practice 15(2): 261-272.

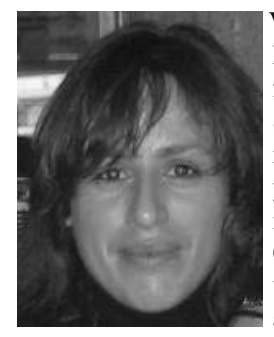

Valérie Dos Santos Martins was born in Troyes, France, in 1976. She graduated in mathematics from the University of Orléans, France, in 2001. She received the Ph.D degree in 2004 in applied mathematics from the same university. After one year at the MAPMO Laboratory of Mathematics in Orléans as an ATER, she was a post-doc at the CESAME/INMA laboratory of the Catholic University of Louvain, Belgium. Currently, she is an assistant professor in the LAGEP laboratory, University of Lyon 1. Her present research interests include nonlinear control theory, perturbations theory of operators and semigroups, spectral theory and control of nonlinear partial differential equations as well as Hamiltonian systems.

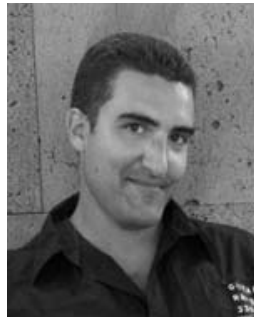

Mickael Rodrigues was born in Essey-lésNancy, France, in 1979. He received his Ph.D. degree in automatic control from the Department of Automatic Control of the Henri Poincaré University of Nancy 1 in 2005. Since 2006, he has been an associate professor at the Claude Bernard University of Lyon 1 with the Automatic and Process Control Laboratory (LAGEP), France. His current research interests are focused on model-based fault diagnosis, fault tolerant control, multi-models, LPV systems, singular/descriptor systems, observers, stability and LMIs.

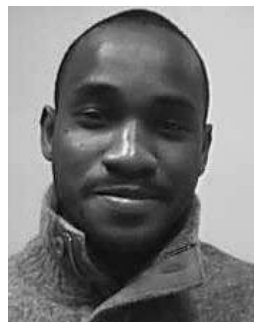

Mamadou Diagne was born in Senegal in 1980 He graduated with an M.Sc. degree from the Claude Bernard University of Lyon. He is currently a Ph.D. student at the LAGEP laboratory, Claude Bernard University of Lyon. His present research interests include linear control theory and Hamiltonian systems.

Received: 21 July 2011

Revised: 26 June 2012 